\title{
Long non-coding RNA ZFAS1 is a major regulator of epithelial-mesenchymal transition through miR-200/ZEB1/E-cadherin, vimentin signaling in colon adenocarcinoma
}

\author{
Stephen J. O'Brien', Casey Fiechter ${ }^{1}$, James Burton', Jacob Hallion', Mason Paas', Ankur Patel', Ajay Patel', \\ Andre Rochet ${ }^{1}$, Katharina Scheurlen ${ }^{1}$, Sarah Gardner ${ }^{1}$, Maurice Eichenberger ${ }^{1}$, Harshini Sarojini ${ }^{1}$, Sudhir Srivastava $\mathbb{D}^{2,3}$, \\ Shesh Rai ${ }^{2}$, Theodore Kalbfleisch $\mathbb{1}^{4}{ }^{4}$, Hiram C. Polkrr ${ }^{1}$ and Susan Galandiuk (i)
}

\begin{abstract}
Colon adenocarcinoma is a common cause of cancer-related deaths worldwide. Epithelial-mesenchymal transition is a major regulator of cancer metastasis, and increased understanding of this process is essential to improve patient outcomes. Long non-coding RNA (InCRNA) are important regulators of carcinogenesis. To identify IncRNAs associated with colon carcinogenesis, we performed an exploratory differential gene expression analysis comparing paired colon adenocarcinoma and normal colon epithelium using an RNA-sequencing data set. This analysis identified IncRNA ZFAS1 as significantly increased in colon cancer compared to normal colon epithelium. This finding was validated in an institutional cohort using laser capture microdissection. ZFAS1 was also found to be principally located in the cellular cytoplasm. ZFAS1 knockdown was associated with decreased cellular proliferation, migration, and invasion in two colon cancer cell lines (HT29 and SW480). MicroRNA-200b and microRNA-200c (miR-200b and miR-200c) are experimentally validated targets of ZFAS1, and this interaction was confirmed using reciprocal gene knockdown. ZFAS1 knockdown regulated ZEB1 gene expression and downstream targets E-cadherin and vimentin. Knockdown of miR-200b or miR-200c reversed the effect of ZFAS1 knockdown in the ZEB1/E-cadherin, vimentin signaling cascade, and the effects of cellular migration and invasion, but not cellular proliferation. ZFAS1 knockdown was also associated with decreased tumor growth in an in vivo mouse model. These results demonstrate the critical importance of ZFAS1 as a regulator of the miR-200/ZEB1/E-cadherin, vimentin signaling cascade.
\end{abstract}

\section{Introduction}

Colorectal cancer is a major cause of cancer-related death worldwide ${ }^{1}$. Although large cohort studies demonstrate that early detection with screening is associated with earlier stage at diagnosis and improved

\footnotetext{
Correspondence: Susan Galandiuk (susan.galandiuk@louisville.edu)

${ }^{1}$ Price Institute of Surgical Research, Hiram C. Polk Jr. MD Department of

Surgery, University of Louisville School of Medicine, Louisville, KY 40202, USA

${ }^{2}$ Department of Bioinformatics and Biostatistics, University of Louisville,

Louisville, KY, USA

Full list of author information is available at the end of the article

Edited by Ivano Amelio
}

survival, many patients still present with advanced stage (Stage III or IV) disease ${ }^{2}$. Increased understanding of the complex processes involved in tumor metastasis are critical to identifying potential treatment targets and improving patient outcomes.

Epithelial-mesenchymal transition (EMT) is a normal part of embryological development but is also a well characterized and critical part of tumor metastasis ${ }^{3}$. Colorectal cancers that demonstrate a mesenchymal phenotype have worse clinical outcomes compared to those with an epithelial phenotype ${ }^{4}$. EMT is a reversible

\section{(c) The Author(s) 2021}

(c) (i) Open Access This article is licensed under a Creative Commons Attribution 4.0 International License, which permits use, sharing, adaptation, distribution and reproduction c. in any medium or format, as long as you give appropriate credit to the original author(s) and the source, provide a link to the Creative Commons license, and indicate if changes were made. The images or other third party material in this article are included in the article's Creative Commons license, unless indicated otherwise in a credit line to the material. If material is not included in the article's Creative Commons license and your intended use is not permitted by statutory regulation or exceeds the permitted use, you will need to obtain permission directly from the copyright holder. To view a copy of this license, visit http://creativecommons.org/licenses/by/4.0/. 
process whereby cells lose key morphological and phenotypic epithelial features and gain mesenchymal features. The process is regulated by both genetic and epigenetic factors, such as non-coding RNA regulation. Elucidation of such factors specific to colorectal cancer are needed to develop more sophisticated screening tools and targeted therapies, thereby improving patient outcomes.

Long-non coding RNA (lncRNA) are a class of molecules that have recently been described to have a major role in cancer signaling ${ }^{5}$. They mediate their effect through a number of different mechanisms of action. The molecular decoy, or competitive endogenous RNA mechanism, is frequently described. With this mechanism, lncRNAs can competitively bind molecules such as microRNA (miRNA), and prevent them from mediating their effect on downstream gene signaling. ZFAS1 is a lncRNA on chromosome 20 and has been examined in different cancers but variable results have been reported ${ }^{6}$. A recent review described that the majority of studies report that ZFAS1 has an oncogenic role, but ZFAS1 has a tumor suppressor role in breast cancer, and conflicting mechanisms have been reported in hepatocellular cancer ${ }^{6}$. It is important to examine the role of lncRNAs in individual cancer types. The miR-200 family is a well characterized regulator of EMT in cancer that has been demonstrated in a number of in vitro and human studies ${ }^{4}$. The miR-200 family is a group of five miRNAs and its core mechanism is regulated through a double-negative feedback loop with ZEB1/ZEB2 expression ${ }^{7}$.

In this study, we describe the identification and validation of IncRNA ZFAS1 as having an important role in colon cancer progression using a large RNA-seq data set and institutional clinical samples. ZFAS1 was increased in expression in CRC tissue compared to normal colon epithelium across all data sets examined. ZFAS1 knockdown was associated with decreased cellular proliferation, migration, and invasion. In examining the mechanism of action, ZFAS1 was able to regulate Zinc-finger E-box binding homeobox 1 (ZEB1) expression by altering miR$200 \mathrm{~b}$ and miR-200c signaling. Therefore, ZFAS1 may be a potential future therapeutic target.

\section{Results}

ZFAS1 is a highly differentially expressed IncRNA in colon adenocarcinoma compared to normal colon epithelium

Using the colon adenocarcinoma TCGA data set, we identified 40 patients with paired colon adenocarcinoma and normal colon epithelium RNA-seq data files. A differential gene expression analysis was performed to identify dysregulated lncRNAs between the paired cancer and normal colon epithelium samples. Using a log fold change of $>1.5$ or $<-1.5$, and a false discovery rate $<0.05$, 348 lncRNAs were found to be differentially expressed (Supplementary Fig. 1).
To narrow the lncRNAs for further investigation, the Cancer Cell Line Encyclopedia was interrogated for colon cancer cell lines with robust IncRNA expres$\operatorname{sion}^{8}$. IncRNAs were also selected on the basis of having experimentally validated lncRNA-miRNA interactions on the DIANA lncBASE platform, as well as reports in the literature ${ }^{9}$. From this, seven IncRNA were selected for further investigation: FAM83H-AS1, PVT1, UCA1, H19, FER1L4, GAS5, and ZFAS1.

Previous studies have shown that the tumor microenvironment can skew data analysis by "contaminating" gene expression in macrodissected tumor samples ${ }^{10,11}$. Therefore, laser capture microdissection was used to investigate IncRNA expression in 23 patients with paired colon adenocarcinoma and normal colon epithelium samples (Table 1, Fig. 1a). Three of the seven lncRNAs (PVT1, GAS5, and ZFAS1) were significantly upregulated and two (FAM83H-AS1 and UCA1) were significantly downregulated in colon adenocarcinoma compared to paired normal colon epithelium samples (Fig. 1b). ZFAS1 was the most significantly increased lncRNA in our validated data set and was therefore selected for further investigation (Fig. 1c). ZFAS1 was significantly increased in expression in three separate sequencing data sets compared to normal tissue (all $p<0.05$ ) (Fig. $1 \mathrm{~d}-\mathrm{f}$ ). As expected, increased ZFAS1 expression was associated with both copy number gain and amplification (Fig. 1g). There was, however, no difference in the overall survival of the TCGA clinical cohort between high and low ZFAS1 expression (Fig. 1h).

Table 1 Clinical details of patients from the University of Louisville Biorepository.

\begin{tabular}{lc}
\hline Variable & $\mathbf{N}=\mathbf{2 3} \mathbf{N}$ (\%) \\
\hline Age at diagnosis (years) (median, interquartile range) & $70(61-77)$ \\
Gender & \\
Male & $16(70)$ \\
Female & $7(30)$ \\
Race & \\
Caucasian & $17(74)$ \\
African-American & $6(26)$ \\
AJCC tumor stage & \\
Stage I & $7(30)$ \\
Stage II & $6(26)$ \\
Stage III & $5(22)$ \\
Stage IV & $5(22)$ \\
\hline
\end{tabular}




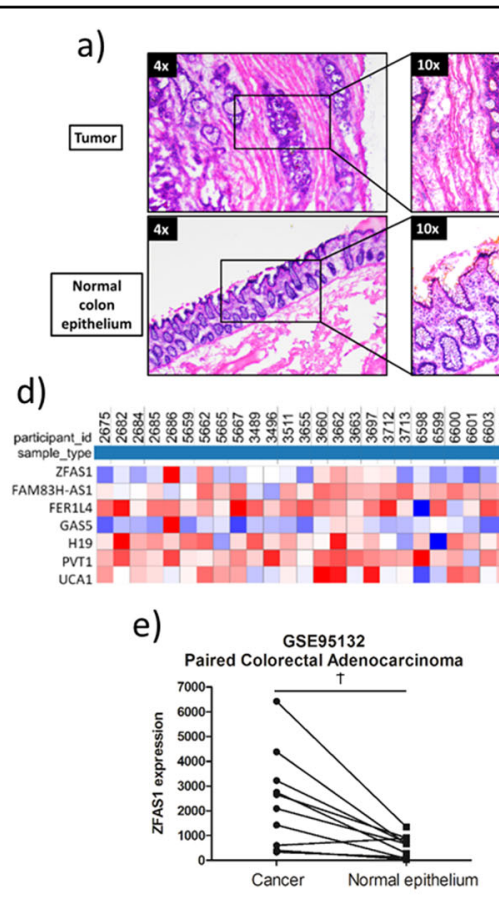

h)

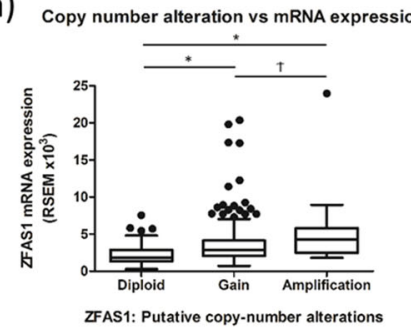

d)

f) b)

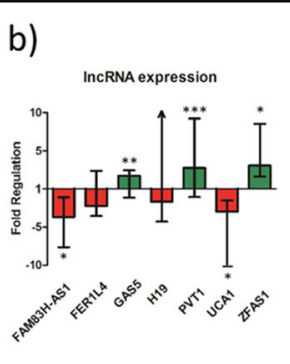

c)

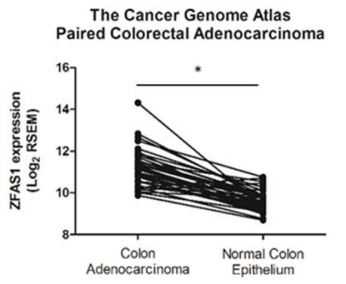

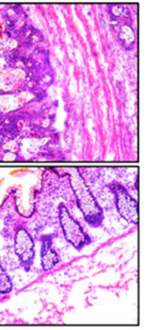
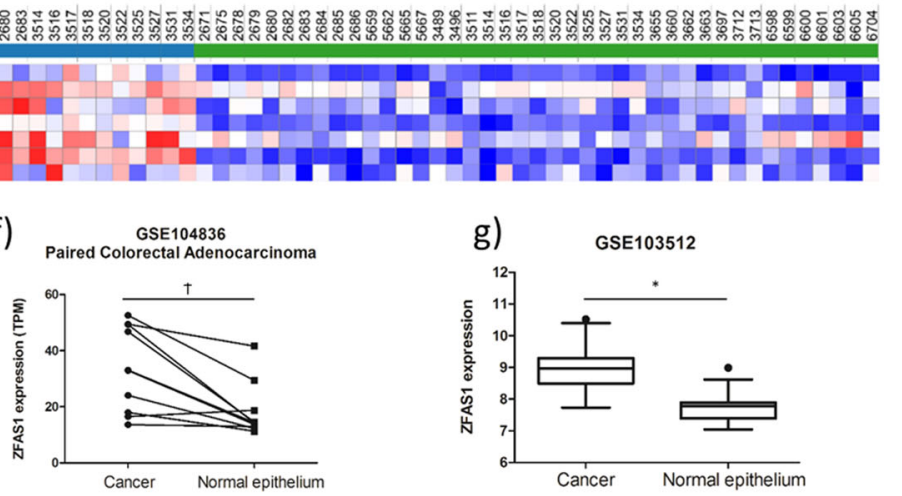

g) GSE103512

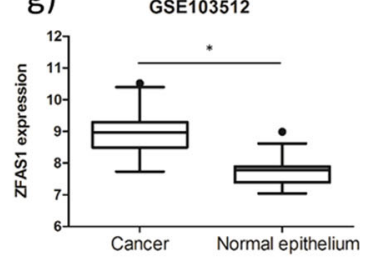

i)

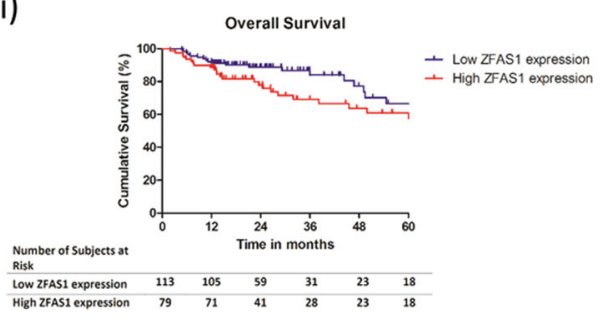

Fig. 1 Characterization of IncRNA expression in the TCGA data set and in an institutional data set, with a specific focus on ZFAS1.

a Example of a hematoxylin and eosin slide that was used as a guide for laser capture microdissection of the tissue from the University of Louisville Biorepository. $\mathbf{b}$ Fold regulaton of each of the seven selected IncRNAs examined by RTgPCR from LCM tissue from the University of Louisville Biorepository; FAM83H-AS1 (FR $\left.=-3.68,{ }^{*} p<0.001\right)$, FER1L4 ( FR $\left.=-2.22 p=0.12\right)$, GAS5 (FR $\left.=1.70,{ }^{* *} p=0.021\right)$, H19 (FR $\left.=-1.67, p=0.84\right)$, PVT1 $\left(\mathrm{FR}=2.75,{ }^{* * *} p=0.003\right), \mathrm{UCA} 1$ (FR $\left.=-4.85,{ }^{*} p<0.001\right), \mathrm{ZFAS1}$ (FR $\left.=3.06,{ }^{*} p<0.001\right)$, $(n=23$ paired samples). c Scatter plot of ZFAS1 expression in colon cancer compared to paired normal colon epithelium examined by RTqPCR from LCM tissue from the University of Louisville Biorepository ( $n=$ 23 paired institutional samples) ( $F C=3.06,{ }^{*} p<0.001$ ). $\mathbf{d}$ Heatmap comparing the differential expression (RSEM quantification) between paired colon adenocarcinoma and normal colon epithelium of the seven IncRNAs using RNAseq expression data from the Cancer Genome Atlas ( $n=40$ paired samples). e Increased ZFAS1 expression in cancer compared to paired normal colon epithelium in the GEO GSE95132 RNA-seq dataset ( $n=10$ paired samples, RNA-seq expression, units =TPM) ( $\left.{ }^{\top} p=0.009\right)$. $\mathbf{f}$ Increased expression of ZFAS1 in cancer compared to paired normal colon epithelium in the GEO GSE104836 RNA-seq dataset ( $n=10$ paired samples, units = normalized RNAseq expression) ( ${ }^{\top} p=0.009$ ). $\mathbf{g}$ Increased expression of ZFAS1 in colon cancer compared to unpaired colon epithelium in the GEO GSE103512 array dataset $(n=70$ Affymetrix array expression, units $=$ normalized Affymetrix array) ( $\left.{ }^{*} p<0.001\right)$. $\mathbf{h}$ There is increased ZFAS1 expression with copy number gain and copy number amplification compared to diploid in the RNAseq TCGA data set $(n=388)\left({ }^{*} p<0.001,{ }^{\top} p<0.009\right)$. $\mathbf{i}$ There is no difference in the overall clinical patient survival between high and low ZFAS1 expression in the RNAseq TCGA data set $(n=192)$ (RNA-seq expression data dichotomized on basis of median expression) ( $p=0.11)$. All RTgPCR experiments were run in duplicate.

\section{ZFAS1 knockdown is associated with a less aggressive phenotype}

ZFAS1 was principally located in the cellular cytoplasm of all three cell lines (Fig. 2a). There was robust ZFAS1 knockdown in all three cell lines (all $>70 \%$ and $p<0.05)$, without a decrease in cellular viability compared to non-target siRNA (all $p>0.05$ ) (Fig. 2b, c). ZFAS1 knockdown was associated with decreased cellular proliferation (Fig. 2d, e) in the HT29 and SW480 cell lines. There was, however, no difference in Caco2 cellular proliferation comparing ZFAS1 knockdown to non-target siRNA, which is likely due to the inherent slow doubling time of the Caco2 cell line ${ }^{12}$. Therefore, for further functional experiments, we focused on the HT29 (epithelial-like) and the SW480 cell line (mesenchymal-like). As such, ZFAS1 knockdown was 
a)
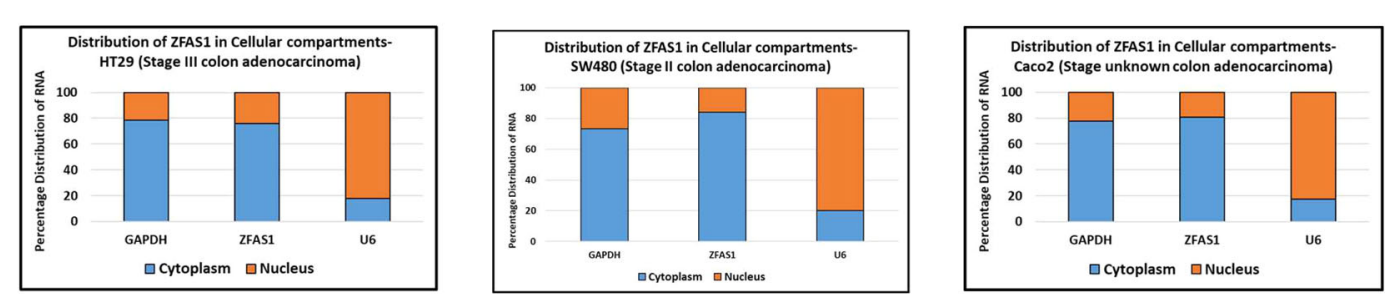

b)

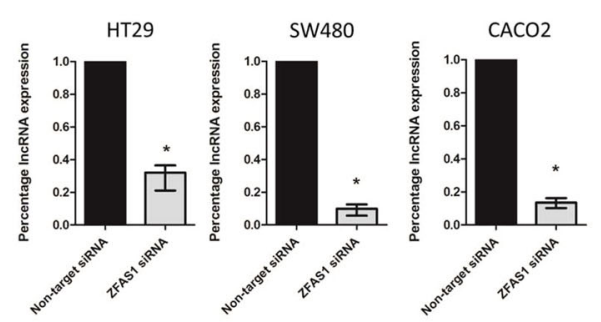

c)
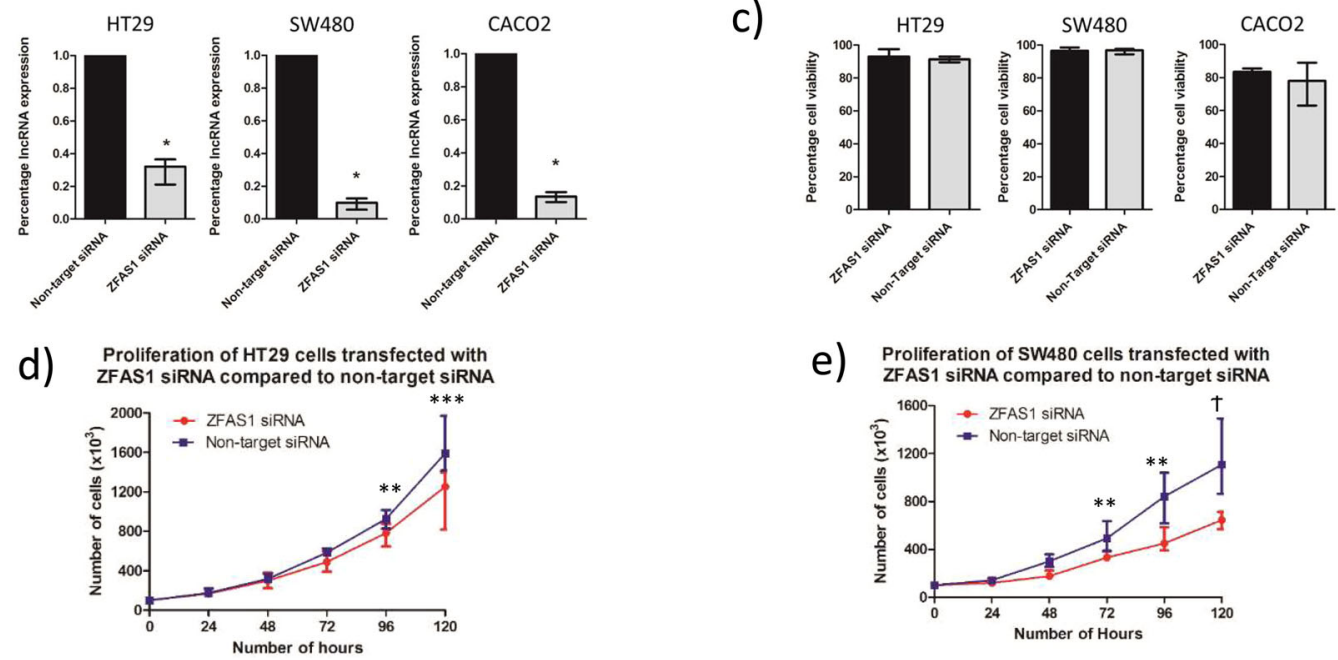

e) Proliferation of SW480 cells transfected with

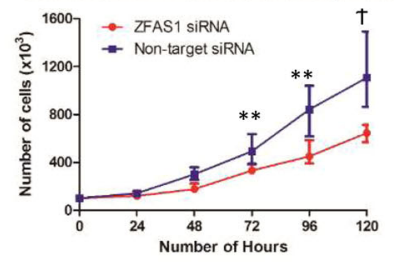

f)

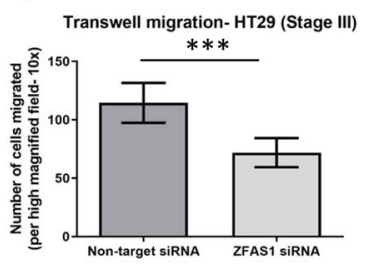

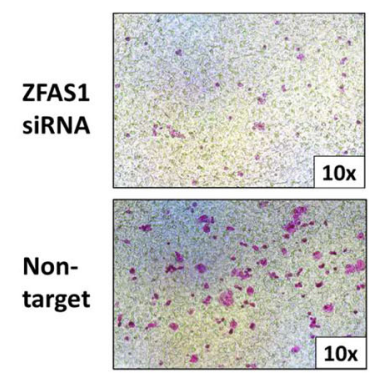

h)
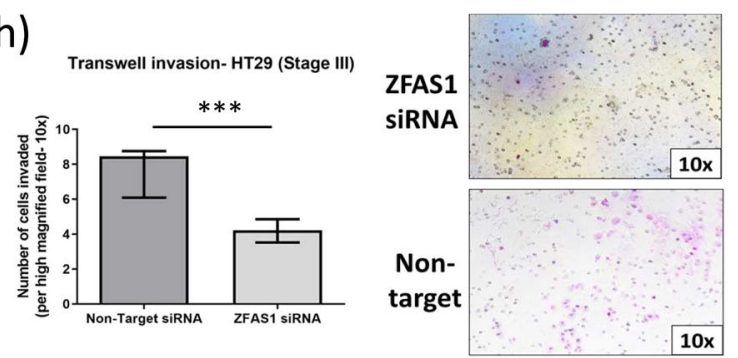

j)

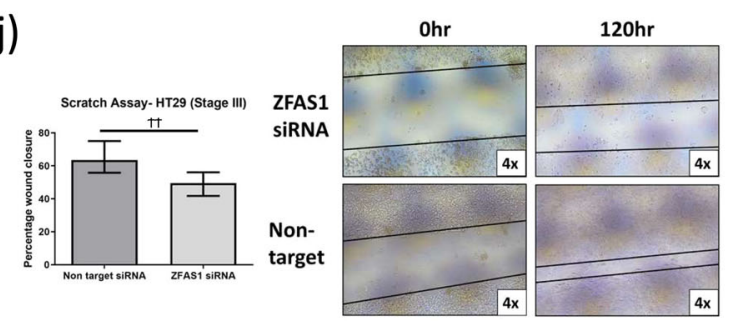

g)

Transwell migration- SW480 (Stage II)

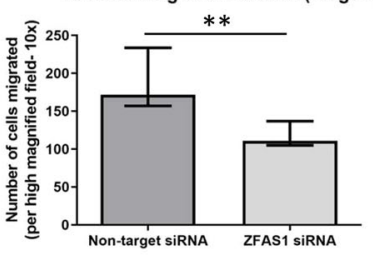

ZFAS1 SIRNA

Nontarget

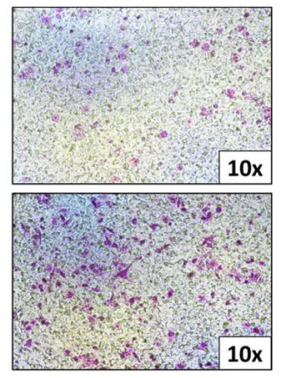

i)

Transwell invasion- SW480 (Stage II)
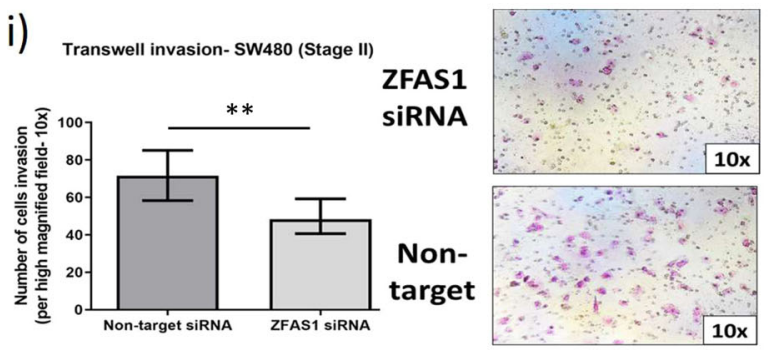

k)

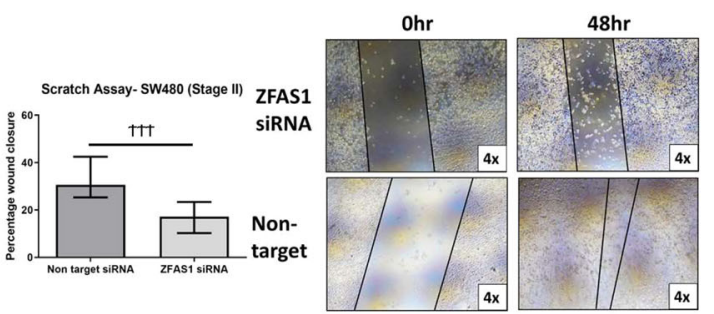

Fig. 2 (See legend on next page.) 
(see figure on previous page)

Fig. 2 Characterization of ZFAS1 function in vitro. a ZFAS1 is principally located in the cytoplasm, compared to the nucleus, measured by RTqPCR in all three colon cancer cell lines (HT29: 76\% vs. $24 \%, p=0.02$, SW480: $84 \%$ vs. $16 \%, p=0.03$, Caco2: $77 \%$ vs. $23 \%, p=0.005, n=6$ replicates). b RTqPCR shows efficient knockdown of ZFAS1 with ZFAS1 siRNA in all three cell lines compared to non-target siRNA (all $>70 \%$, all ${ }^{*} p<0.05, n=6$ replicates). $\mathbf{c}$ There is no decrease in the cell viability (percentage of viable cells compared to all counted cells) with ZFAS1 knockdown compared to cells transfected with non-target siRNA ( $n=6$ replicates). $\mathbf{d}$ There is significant decrease in the cellular proliferation with ZFAS1 knockdown in the HT29 cell lines, by examining the doubling time ( $34.3 \mathrm{~h}$ vs. $30.6 \mathrm{~h}, p=0.046)$, the individual counts at $96 \mathrm{~h}(* * p=0.03)$, and the counts at $120 \mathrm{~h}$ $\left({ }^{* *} p=0.02\right)(n=6$ replicates). e There is significant decrease in the cellular proliferation with ZFAS1 knockdown in the SW480 cell lines, by examining the doubling time ( $42.5 \mathrm{~h}$ vs. $31.5 \mathrm{~h}, p=0.01)$, and the individual counts at $72 \mathrm{~h}\left({ }^{* *} p=0.03\right), 96 \mathrm{~h}\left({ }^{* *} p=0.03\right)$, and $120 \mathrm{~h}\left({ }^{\top} p=0.01\right)(n=6$ replicates). $\mathbf{f}$ There is significantly decreased migration of cells with ZFAS1 knockdown in the HT29 cell line $\left({ }^{* * *} p=0.02, n=6\right.$ replicates) The panels on the right show representative $\times 10$ magnification fields of the HT29 cell line transwell migration assay (top panel ZFAS1 knockdown, bottom panel non-target siRNA). $\mathbf{g}$ There is significantly decreased migration of cells with ZFAS1 knockdown in the SW480 cell line $(* * p=0.03, n=6$ replicates). The panels on the right show representative $\times 10$ magnification fields of the SW480 cell line transwell migration assay (top panel ZFAS1 knockdown, bottom panel non-target siRNA). $\mathbf{h}$ There is significantly decreased invasion of cells with ZFAS1 knockdown in the HT29 cell line $\left({ }^{* * *} p=0.02, n=6\right.$ replicates) The panels on the right show representative $\times 10$ magnification fields of the HT29 cell line transwell invasion (top panel ZFAS1 knockdown, bottom panel non-target siRNA). $\mathbf{i}$ There is significantly decreased invasion of cells with ZFAS1 knockdown in the SW480 cell line $\left({ }^{* *} p=0.03, n=6\right.$ replicates). The panels on the right show representative $\times 10$ magnification fields of the SW480 cell line transwell invasion (top panel ZFAS1 knockdown, bottom panel non-target siRNA). $\mathbf{j}$ There was a significantly slower scratch closure of the HT29 cell line with ZFAS1 knockdown compared to control ( ${ }^{\top \top} p=0.004, n=11$ replicates). The panels on the right show representative $\times 4$ magnification fields of the HT29 scratch assay (top panels scratch closure following ZFAS1 knockdown at time 0 and at $120 \mathrm{~h}$, bottom panels scratch closure with non-target siRNA at time 0 and at 120 h). $\mathbf{k}$ There was a signficiantly slower scratch closure of the SW480 cell lines with ZFAS1 knockdown compared to control $\left({ }^{+\top+} p<0.001, n=6\right.$ replicates). The panels on the right show representative $\times 4$ magnification fields of the SW480 scratch assay (top panels scratch closure following ZFAS1 knockdown at time 0 and at $48 \mathrm{~h}$, bottom panels scratch closure with non-target siRNA at time 0 and at $48 \mathrm{~h}$ ).

Table 2 List of IncRNA-miRNA interactions.

\begin{tabular}{llll}
\hline IncRNA & miRNA & $\begin{array}{l}\text { Literature } \\
\text { citation }\end{array}$ & $\begin{array}{l}\text { Method of experimental } \\
\text { verification }\end{array}$ \\
\hline ZFAS1 & miR-200b & Liu G. et al. ${ }^{13}$ & RNA immunoprecipitation \\
& Zhang F et al. ${ }^{14}$ & RNA pulldown assay \\
miR-200c & Liu G. et al. ${ }^{13}$ & RNA immunoprecipitation \\
miR-150 & Xia B. et al. ${ }^{15}$ & Luciferase reporter assay \\
& Li T. et al. ${ }^{16}$ & RNA pulldown assay \\
& Wu T. et al. ${ }^{17}$ & RNA pulldown assay \\
& Chen $X$ et al. ${ }^{18}$ & RNA pulldown assay \\
miR-484 & Xie $S$ et al. ${ }^{19}$ & RNA immunoprecipitation \\
miR-27a & Ye Y et al. ${ }^{20}$ & RNA pulldown assay \\
& & Luciferase reporter assay
\end{tabular}

associated with decreased transwell migration and invasion (Fig. 2f-i) and a slower scratch assay closure (Fig. 2j, k) in the HT29 and SW480 cell lines. As expected, both transwell assays and scratch closure demonstrated slower migration of the HT29 cell line compared to the SW480 cell line. This is likely due to the HT29 cell line having an epithelial-like Consensus Molecular Subtype 3 (CMS3) compared to the mesenchymal-like CMS4 of the SW480 cell line ${ }^{12}$.

\section{Investigation of experimentally validated miRNA targets of ZFAS1}

As previously mentioned, there are experimentally validated lncRNA-miRNA interactions for ZFAS1 (Table 2) (refs. ${ }^{13-20}$ ). Following ZFAS1 knockdown, expression of both miR-200b and miR-200c was significantly increased compared to non-target siRNA (Fig. 3a, b). The lncRNAmiRNA binding sites from the DIANA lncBASE platform are shown in Fig. 3c, d. ZFAS1 was inversely correlated with miR-200c (Spearman $r=-0.120, p=0.09$ ), but not inversely correlated with miR-200b (Spearman $r=$ $-0.030, p=0.65$ ) in the TCGA data set (Fig. 3e, f). Although ZFAS1 does not inversely correlate with miR200b, lncRNA and miRNA expression are dependent on a number of different upstream regulatory molecules ${ }^{21}$. We have previously reported the adverse role of low miR-200b and miR-200c expression with overall survival ${ }^{22}$. Both mir-200b and miR-200c are increased in colorectal cancer compared to paired normal colon epithelium. In addition, both miR-200b and miR-200c are decreased in expression in Stage III/IV compared to Stage I/II colon cancer. Furthermore, transfection with either miR-200b or miR200c mimics leads to decreased ZFAS1 expression in the both the HT29 and SW480 cell lines (Fig. 3g-j). The direct binding interaction between ZFAS1 and miR-200b between miR-200c has been shown previously through both RNA immunoprecipitation ${ }^{15}$ and an RNA pulldown assay $^{16}$. This defined interaction was examined for subsequent mechanistic and functional assays.

ZFAS1 knockdown is associated with changes in the ZEB1 and is mediated through miR-200b and miR-200c signaling

Previous studies have demonstrated the major role of the miR-200 family in cancer signaling, particularly in EMT regulation ${ }^{4}$. Transfection with either ZFAS1 siRNA, miR-200b, or miR-200c mimics leads to ZEB1 mRNA knockdown in both the HT29 and SW480 cell lines 


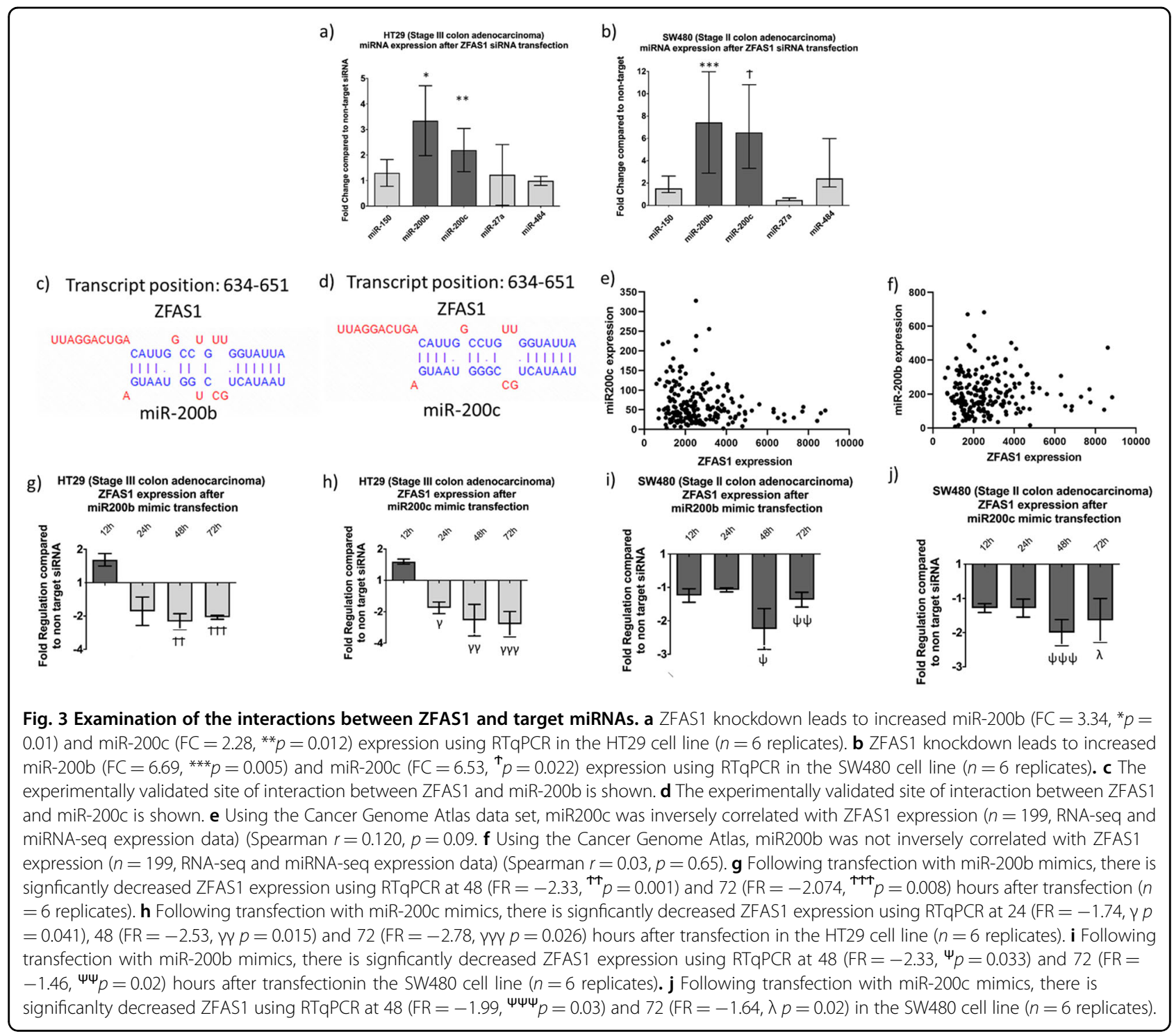

(Fig. 4a, b). ZFAS1 knockdown, in turn, leads to decreased ZEB1 protein expression in both cell lines (Fig. 4c, d). Furthermore, dual knockdown of ZFAS1 and miR-200b or ZFAS1 and miR-200c reversed this effect on ZEB1 expression in both the HT29 and SW480 cell lines. As miR-200b and miR-200c act directly on ZEB1, pronounced effects are seen on ZEB1 expression with miRNA mimics and antagomirs.

Increased ZFAS1 expression and decreased miR-200b or miR-200c expression is associated with adverse clinical outcomes, and ZFAS1 mediates its effect on the E-cadherin vimentin signaling through miR-200b and miR-200c signaling

As previously mentioned, ZFAS1 expression alone is not associated with adverse clinical outcomes. However, when ZFAS1 expression is stratified by both miR-200b
(Fig. 5a, b) and miR-200c expression (Fig. 5c, d), high ZFAS1 expression is associated with worse overall survival in the setting of low miR-200b $(p=0.018)$ and low miR$200 \mathrm{c}(p=0.048)$ expression. This suggests that patients with high ZFAS1 expression and low miR-200b or low miR-200c expression have a particularly poor genomic signature. As hypothesized, downstream of ZEB1, ZFAS1 knockdown leads to increased E-cadherin expression as well as decreased vimentin protein expression (Fig. $5 \mathrm{e}-\mathrm{h}$ ) in both the HT29 and SW480 cell lines. This regulatory effect on E-cadherin and vimentin expression was attenuated with dual transfection of ZFAS1 and miR-200b or ZFAS and miR-200c in both the HT29 and SW480 cell lines. Interestingly, we did not observe an increase in the E-cadherin expression with miR-200b or miR-200c mimic transfection. There is low endogenous expression of ZEB1 in the HT29 cell line, and this may explain the inability of 

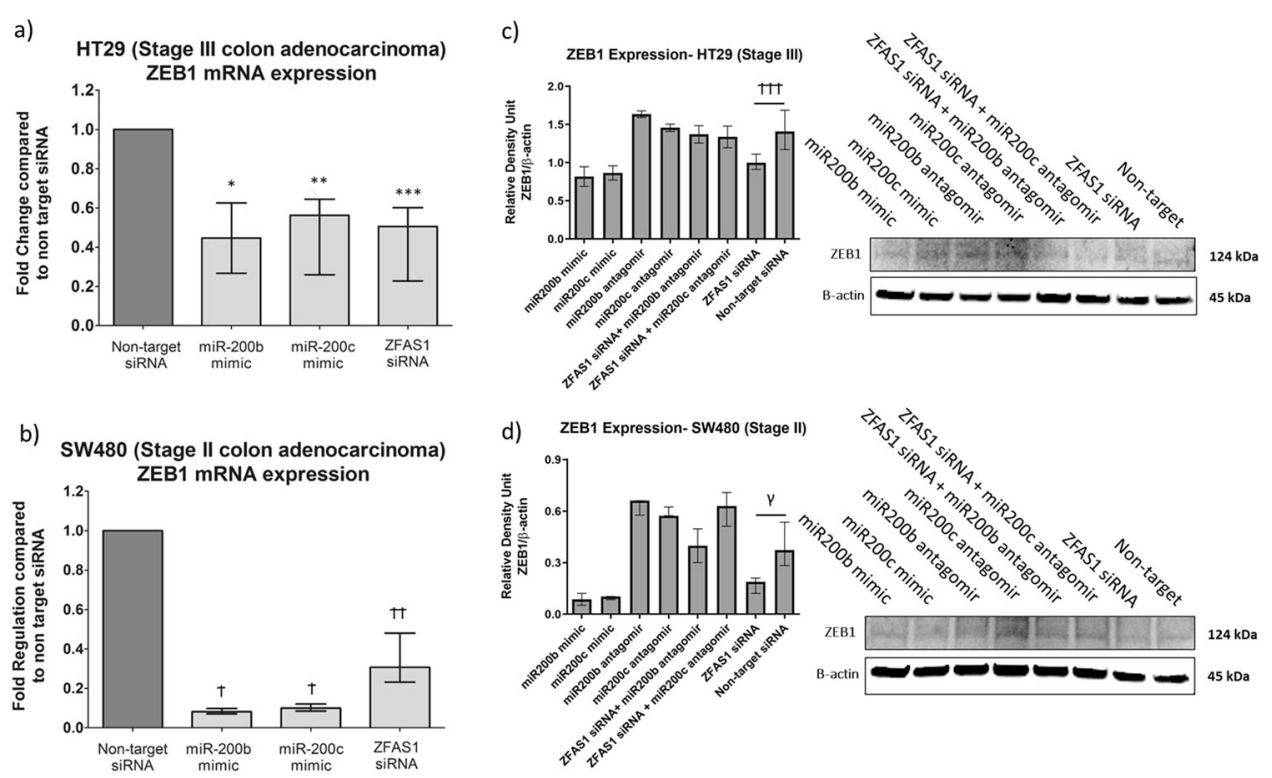

Fig. 4 ZFAS1 knockdown is associated with changes in ZEB1 expression. This effect is reversed by dual knockdown of ZFAS1 and miR200b or with ZFAS1 and miR200c. a In the HT29 cell line, ZFAS1 knockdown ( $\left.F R=-2.68,{ }^{*} p=0.031\right)$, miR-200b mimics ( $F R=-3.19$, $\left.{ }^{* *} p=0.03\right)$, or miR-200c mimics ( $F R=-1.82,{ }^{* * *} p=0.04$ ) leads to decreased ZEB1 mRNA expression using RTqPCR ( $n=6$ replicates). $\mathbf{b}$ In the SW480 cell line, ZFAS1 knockdwon ( $F R=-3.37,{ }^{\top} p=0.034$ ), miR-200b mimics ( $\left.F R=-12.24,{ }^{\top} p<0.001\right)$, or miR-200c mimics ( $\left.F R=-10.1,{ }^{t+} p<0.001\right)$ knockdown leads to decreased ZEB1 mRNA expression using RTqPCR ( $n=6$ replicates). c There is significantly decreased ZEB1 protein expression with ZFAS1 knockdown $\left({ }^{+\top+} p=0.046\right)$ in the HT29 cell line by Western blotting. This effect was reversed by dual knockdown of either ZFAS1 and miR200b or ZFAS1 and miR200c (both $p>0.05$ ). As expected, miR200b and miR200c antagomirs increased ZEB1 expression, with reciprocal decreased in ZEB1 expression with miR-200b and miR200c mimics ( $n=5$ replicates). $\mathbf{d}$ Similarly, there is significantly decreased ZEB1 protein with ZFAS1 knockdown in the SW480 cell line by Western blotting ( $y p=0.047$ ). This effect was reversed by dual knockdown of either ZFAS1 and miR200b or ZFAS1 and miR200c (both $p>0.05)$. As expected, miR200b and miR200c antagomirs increased ZEB1 expression, with reciprocal decreased in ZEB1 expression with miR-200b and miR200c mimics ( $n=5$ replicates).

miR-200b or miR-200c to indirectly regulat E-cadherin expression. This explanation may also account for the differences seen in vimentin expression in the HT29 cell line.

miR-200b and miR-200c knockdown partially reverse the functional effect of ZFAS1 knockdown on proliferation, migration, and invasion

To validate these signaling cascade results, we performed functional cellular assays with dual knockdown of both ZFAS1 and miR-200b or ZFAS1 and miR-200c. In the HT29 cell line, there was no difference in cellular proliferation with dual knockdown of ZFAS1 and miR$200 \mathrm{~b}$ compared to non-target. However, there was a significant difference in the doubling time of cells with dual knockdown of ZFAS1 and miR-200c compared to non-target (Fig. 6a, Supplementary Table 4). In the SW480 cell line, there was no difference in the doubling time following ZFAS1 and miR-200c knockdown compared to non-target, but there were significant differences in the individual cell counts at $96 \mathrm{~h}(p=0.003)$ and $120 \mathrm{~h}(p=0.02)$. Following knockdown with ZFAS1 and miR-200b, there was a slower doubling time of cells as compared to non-target (Fig. 6b, Supplementary
Table 4). In both cell lines, under both conditions, there was no difference in the transwell migration or invasion compared to non-target (all $p>0.05$ ) (Fig. 6c-f). In the HT29 cell line, there was a significant difference in the scratch closure of cells following dual ZFAS1 and miR200c knockdown compared to non-target $(p=0.02)$, but there was no difference in the dual ZFAS1 and miR$200 \mathrm{~b}$ knockdown. In addition, there was no difference in the scratch closure in either of the SW480 dual knockdown conditions (Fig. 6g, e).

\section{ZFAS1 knockdown is associated with decreased tumor growth in vivo}

Previous experiments describe the clinical relevance and the mechanisms of action of ZFAS1 in colorectal cancer cell lines, but it is important to explore its mechanism of action in vivo. Using a mouse model, ZFAS1 knockdown was associated with decreased tumor volume compared to non-target siRNA (Fig. 7a). A summary of the results of this study are illustrated in Fig. 7b.

\section{Discussion}

We hypothesized that using a RNA-sequencing data set to compare the entire transcriptome of colon adenocarcinoma 


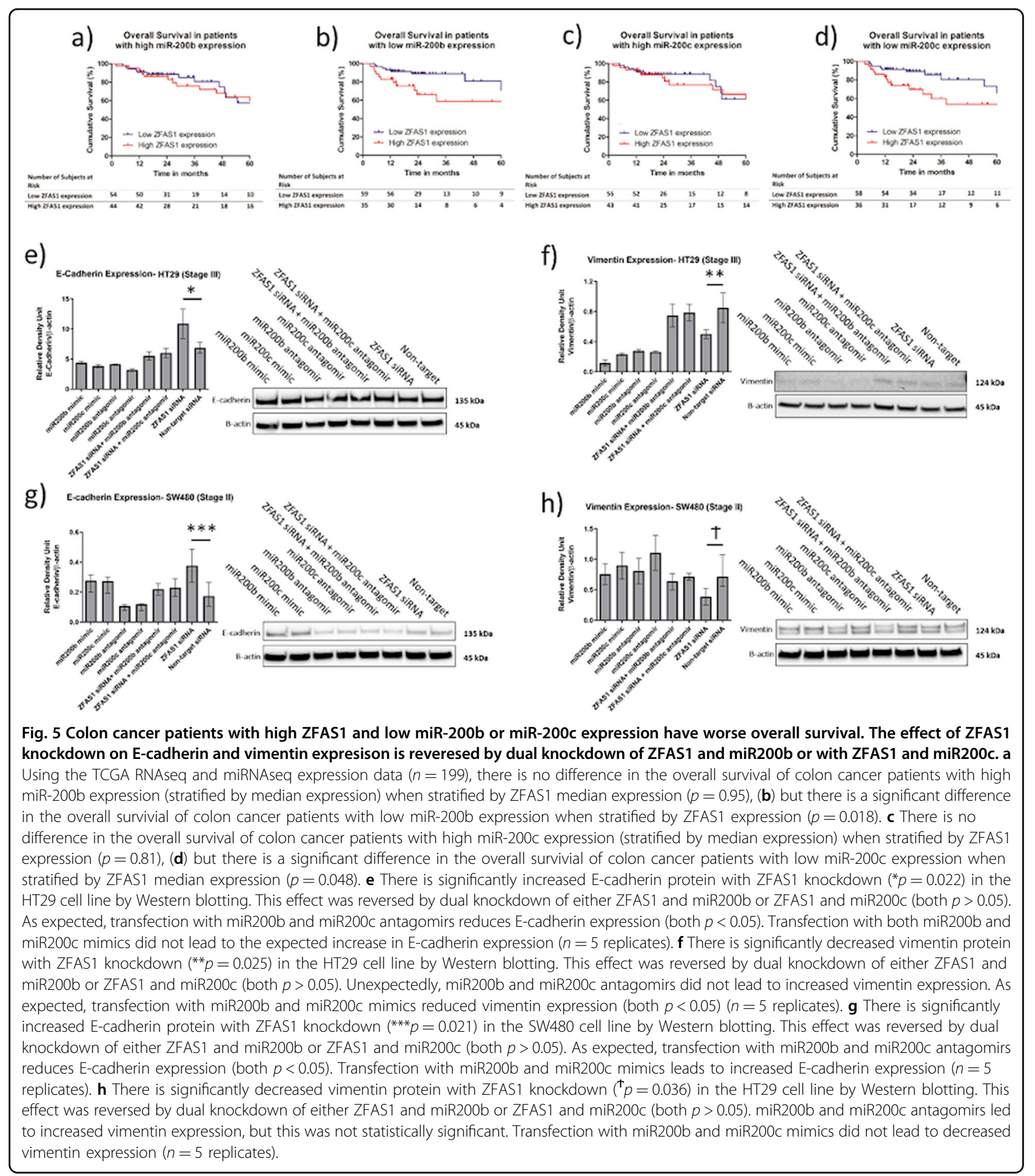

tissue to paired normal colon epithelium tissue would allow us to investigate differentially expressed lncRNAs that are critical in colorectal carcinogenesis. We validated the expression of ZFAS1, identifying it as a major regulator of tumor progression in CRC, through the critical miR-200/ ZEB1/E-cadherin, vimentin signaling cascade.
There are variable results when examining ZFAS1 as a mediator of carcinogenesis in the literature ${ }^{6}$. ZFAS1 has been shown to have a tumor suppressive function in breast cancer by decreasing cellular proliferation and migration $^{23,24}$. In hepatocellular carcinoma, ZFAS1 can have both a tumor suppressive and oncogenic function. 


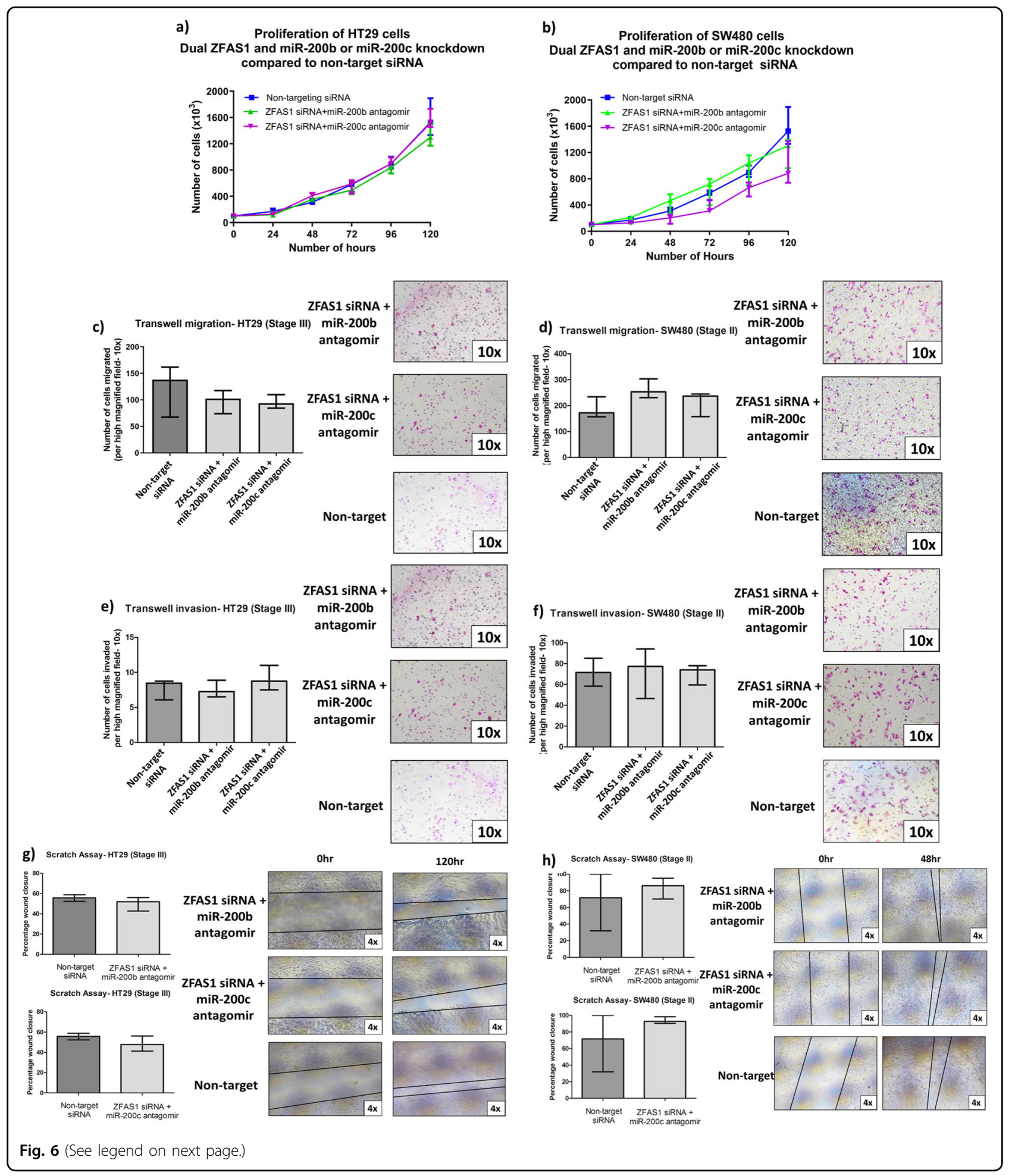

For example, it can act as a tumor suppressor by methylating miR-9 in hepatocellular carcinoma ${ }^{25}$, but can also promote cancer metastasis through miR-150 binding $^{16}$. The majority of studies, however, demonstrate that ZFAS1 has an oncogenic function, many indicating that
ZFAS1 can regulate both proliferation and migration ${ }^{13,15}$. Recently, Chen et al. reported that ZFAS1 could regulate proliferation, migration, and invasion in colorectal can$\operatorname{cer}^{18}$. ZFAS1 could mediate an indirect regulation of VEGFA by directly binding miR-150. The results of our 
(see figure on previous page)

Fig. 6 Knockdown of miR-200b or miR-200c partially reverses the effect on proliferation, but reverses the effect on migration and invasion. a In the HT29 cell line, there is no difference in cell proliferation following dual ZFAS1 and miR-200b knockdown compared to non-target siRNA, when measured by doubling time $(p=0.46)$, but there is more rapid proliferation with dual knockdown of ZFAS1 and miR-200c compared to non-target siRNA ( ${ }^{\circ}$ doubling time: $26.8 \mathrm{~h}$ vs. $30.6 \mathrm{~h}, p=0.01$ ) ( $n=6$ replicates). $\mathbf{b}$ In the SW480 cell line, cell proliferation following dual ZFAS1 and miR-200b knockdown is slower than compared to non-target siRNA (**doubling time: 35.9 vs. 30.5, $p=0.06$ ), but there was no difference in proliferation with dual knockdown of ZFAS1 and miR-200c compared to non-target siRNA ( $p=0.55)$ ( $n=6$ replicates). c, $\mathbf{d}$ In the HT29 and SW480 cell lines, there is no difference in the transwell migration with both ZFAS1 and miR-200b or ZFAS1 andmiR-200c knockdown compared to nontarget siRNA (all $p>0.05)$ ( $n=6$ replicates), The panels on the right of each figure show representative $\times 10$ magnification fields of the HT29 and SW480 cell lines transwell migration assay (top panel ZFAS1 knockdown + miR-200b antagomir, middle panel ZFAS1 knockdown + miR-200c antagomir, bottom panel non-target siRNA). e, $\mathbf{f}$ In the HT29 and SW480 cell lines, there was no difference in the transwell invasion with both ZFAS1 and miR-200b or ZFAS1 and miR-200c knockdown compared to non-target siRNA (all $p>0.05)$ ( $n=6$ replicates). The panels on the right of each figure show representative $\times 10$ magnification fields of the HT29 and SW480 cell lines transwell migration assay (top panel ZFAS1 knockdown + miR200b antagomir, middle panel ZFAS1 knockdown + miR-200c antagomir, bottom panel non-target siRNA). $\mathbf{g}$ In the HT29 cell line, there was no difference in scratch closure in both ZFAS1 and miR-200b or ZFAS1 and miR-200c knockdown compared to non-target siRNA (all $p>0.05)(n=6$ replicates). The panels on the right show representative $\times 4$ magnification fields of the HT29 scratch assay (top panels scratch closure following dual ZFAS1 and miR-200b knockdown at time 0 and at $120 \mathrm{~h}$, middle panels scratch closure following dual ZFAS1 and miR-200c knockdown at time 0 and $120 \mathrm{~h}$, bottom panels scratch closure with non-target siRNA at time 0 and at $120 \mathrm{~h}$ ). $\mathbf{h}$ In the SW480 cell line, there was no difference in scratch closure in both ZFAS1 and miR-200b or ZFAS1 and miR-200c knockdown compared to non-target siRNA (all $p>0.05)(n=6$ replicates). The panels on the right show representative $\times 4$ magnification fields of the SW480 scratch assay (top panels scratch closure following dual ZFAS1 and miR-200c knockdown at time 0 and at $48 \mathrm{~h}$, middle panels scratch closure following dual ZFAS1 and miR-200c knockdown at time 0 and $48 \mathrm{~h}$, bottom panels scratch closure with non-target siRNA at time 0 and at $48 \mathrm{~h}$ ).

a)

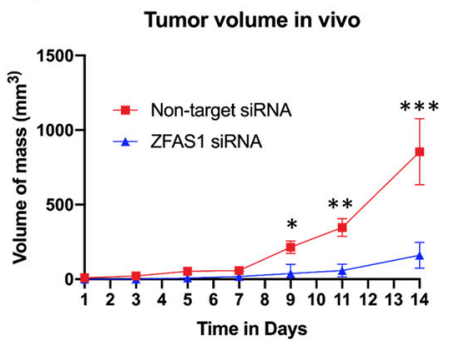

b)

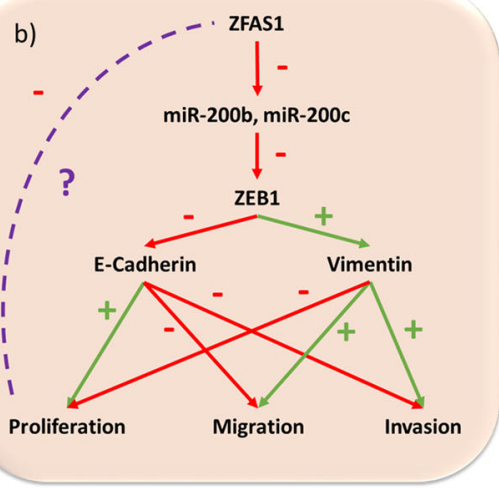

Fig. 7 ZFAS1 knockdown is associated with decreased tumor growth in vivo compared to not-target siRNA. a ZFAS1 knockdown was associated with decreased tumor volume in a mouse xenograft model compared to non-target siRNA, with significant differenes observed from day 3 after mass was first identified in each mouse. The differences were most pronounced from day 7 up until day 14 when the experiment was terminated $\left(N=4\right.$ replicates) $\left.{ }^{*} p=0.006,{ }^{* *} p<0.001,{ }^{* * *} p=0.024\right)$. b Illustration of the signaling cascade demonstrated by these results.

experiments support the functional data reported by Chen et al, in that ZFAS1 knockdown leads to decreased proliferation and migration. The direct binding interaction between ZFAS1 and the miR-200 family has been shown previously through different mechanistic assays including RNA immunoprecipitation ${ }^{15}$ and RNA pulldown ${ }^{16}$. We have validated this interaction in both colorectal cancer patients and in colorectal cancer cell lines.

Interestingly, we also investigated the interaction with miR-150, but we did not find a reciprocal expression change. This finding contrasts with Chen et al., as they reported that miR-150 had a direct binding interaction with ZFAS1 in the HCT116 cell line. This explanation for this discrepancy is unclear, however, and requires further investigation. Nevertheless, both sets of data support the concept that lncRNAs can have multiple mechanisms of action through different signaling cascades. This attribute has been described as both a positive and negative aspect regarding their potential utility ${ }^{26,27}$.

Non-coding RNAs are being investigated as a small molecule therapeutic strategy for different diseases. There are a number of clinical trials exploring both miRNA and siRNA technology as a therapeutic strategy ${ }^{28}$. For example, a novel small molecule targeting miR-122 has been effective in reducing RNA levels of hepatitis $C$ virus in a phase 2 clinical trial ${ }^{29}$. In the future, lncRNAs and other non-coding RNAs may have a major role in the therapeutic management of different diseases. 
Many studies demonstrate that miRNA can regulate lncRNAs through the competitive endogenous RNA mechanism, but few examine upstream regulators. We report that there is copy number amplification seen with ZFAS1 in the TCGA colon adenocarcinoma cohort. Additionally, transcription factor, SP1, has been shown to transcriptionally increase ZFAS1 in a number of different cancer types. ${ }^{6}$. This suggests that modulating specific transcription factors adds a further layer of complexity when studying the relationship between lncRNAs and protein coding genes. This complex regulatory network could partially explain why we do not find a clear inverse relationship between ZFAS1 and miR200b or miR200c, as a number of different upstream regulators can affect their expression.

The results of these experiments highlight areas requiring further investigation. There is some conflict between the results from the TCGA differential gene expression and our results from laser capture microdissection. The tissue that is used for RNA-seq in the TCGA data is from a macrodissected tumor sample. Therefore, it includes not only cancer cells, but also other cells from the tumor microenvironment ${ }^{10}$. Previous studies have shown that molecules, such as miRNA, can have different expression, depending on the location from which the RNA is extracted from a cancer specimen (e.g., superficial tumor, deep border etc) ${ }^{11,30}$. In addition, cells from within the tumor microenvironment may express or secrete lncRNAs in order to induce an effect on cancer cells $^{31}$. In this case, whole tissue dissection may lead to the false conclusion that the IncRNA is expressed from the cancer cells instead of from cells within the tumor microenvironment. TGF- $\beta$ is a major inducing agent of $\mathrm{EMT}^{32}$, and recent studies have demonstrated that it can be secreted by macrophages and other immune cells in the tumor microenvironment ${ }^{33}$. This could be investigated through the use of transwell chambers or by treating colon adenocarcinoma cell lines with cultured macrophage secretions, as performed by Jahangiri et al. ${ }^{31}$. Although our study demonstrates the role of ZFAS1 signaling through regulation of miR-200b and miR-200c, ZFAS1 interacts with a number of other miRNAs, suggesting further work is required to fully delineate the function of ZFAS1 and other lncRNAs.

There are a number of limitations to this work. As previously mentioned, IncRNAs can mediate an effect on a number of different signaling pathways; however, we focused on a single signaling pathway to help delineate the mechanism of action of ZFAS1. There are five members of the miR-200 family, and we restricted our study to two of these, miR-200b and miR-200c, since they have been experimentally verified to interact with ZFAS1. Although miR-200b, miR-200c, and miR-429 are part of the same functional cluster, we did not examine the role of miR-429, as it was not an experimentally validated target of ZFAS1. Further studies could investigate the role of other members of the miR-200 family in relation to ZFAS1. Although we selected lncRNAs that are increased in expression in colon cancer compared to normal colon epithelium, there are a large number of lncRNAs that are downregulated in expression. Due to the large size of lncRNA, stable transfection is challenging, but some investigators have suggested the use of CRISPR-activation technology to stably increase the expression of an lncRNA in vitro ${ }^{34,35}$. Further experiments examining miRNA, gene expression, and proliferations markers in the xenograft tumors is warranted.

In conclusion, we identified and validated that ZFAS1 can regulate EMT through a reciprocal interaction with both miR-200b and miR-200c. Knockdown of ZFAS1 can lead to a less aggressive phenotype in colon cancer cell lines, reduction in the mesenchymal marker vimentin, and an increase in the epithelial marker E-cadherin. This indicates that ZFAS1 is a major regulator of cancer aggressiveness and could be a target for therapeutic intervention in the future.

\section{Methods}

A detailed methods section is described in the Supplementary Methods

\section{Ethics statement}

This study was approved by the institutional review board of the University of Louisville and the Institutional Animal Care and Use Committee of the University of Louisville.

\section{The Cancer Genome Atlas and Gene Expression Omnibus (GEO)}

The raw sequencing data for colon adenocarcinoma patients were identified from the Genomic Data Commons portal (https://portal.gdc.cancer.gov/). An R package "edgeR" was used to identify differentially expressed genes between a 40 patient subset that had both colon adenocarcinoma and normal colon epithelium RNA-seq data files ${ }^{36,37}$. Heatmap was generated using MORPHEUS (https://software.broadinstitute.org/morpheus).

Two paired data sets (colon cancer tissue and normal colon mucosa from the same patient) GSE104836 $(N=$ $10)$ and GSE95132 $(N=10)$, and one unpaired data set (colon cancer tissue and normal colon mucosa from different patients), GSE103512 $(N=70)$, were downloaded from the GEO database.

\section{Bioinformatics analysis}

Each lncRNA was entered to the bioinformatics prediction program DIANA-lncBASE v2.0 (ref. ${ }^{9}$ ). IncRNA targets and signaling pathways were identified using Ingenuity Pathway Analysis (Qiagen, Hilden Germany.) 


\section{Patient samples and laser capture microdissection}

Fresh frozen samples of paired colon adenocarcinoma and normal adjacent colon epithelium from 23 patients were obtained from the University of Louisville Surgical Biorepository. Tissue sections were mounted on negatively-charged slides and stained using the Applied Biosystems Arcturus Staining Kit (Thermo Fisher Scientific, Bedford, MA). The ArcturusXT ${ }^{\mathrm{TM}}$ Laser Capture Microdissection System was used to isolate the tissue of interest. RNA was extracted using the PicoPure ${ }^{\text {TM }}$ RNA Isolation Kit (Thermo Fisher Scientific, Waltham, MA).

\section{Cell lines}

The HT29 (ATCC $^{\circledR}{\text { HTB- } 38^{\text {TM }} \text { ), SW480 }}$ ATCC $^{\circledR}$ CCL-228 ${ }^{\mathrm{TM}}$ ), and $\mathrm{Caco} 2$ (ATCC $^{\circledR}$ HTB- $^{\mathrm{TMM}}{ }^{\mathrm{TM}}$ ) colon adenocarcinoma cell lines were purchased new from the American Type Culture Collection (Manassas, VA). Cell lines were authenticated using Short Tandem Repeat profiling (ATCC, Manassas VA) and tested for mycoplasma using the MycoFluor ${ }^{\mathrm{TM}}$ Mycoplasma Detection Kit (Life Technologies, Carlsbad, CA).

\section{RNA interference}

Small interfering RNA (siRNA) (Dharmacon ${ }^{\mathrm{TM}}$, Lafayette, CO), miRNA mimics (Dharmacon ${ }^{\mathrm{TM}}$ ) and miRNA antagomirs (Life Technologies) were purchased (Supplementary Table 1). All transfections were performed using Dharmafect-1 transfection reagent (Dharmacon ${ }^{\mathrm{TM}}$ ).

\section{RNA extraction}

The Protein and RNA Isolation System ${ }^{\mathrm{TM}}$ (Life Technologies ${ }^{\circledR}$ ) was used to extract nuclear and cytoplasmic compartment RNA. Total RNA extracted using the Qiagen miRNeasy Mini kit (Qiagen). RNA concentration and purity were assessed using Nanodrop ${ }^{\circledR} 2000$ spectrophotometry (Thermo Fisher Scientific).

\section{Real-time quantitative polymerase chain reaction (RT-qPCR)- mRNA and IncRNA quantification}

Complementary DNA (cDNA) was generated using the Superscript $^{\mathrm{TM}}$ IV VILO ${ }^{\mathrm{TM}}$ Master Mix (Life Technologies). qPCR was performed using TaqMan Fast Advanced Master Mix (Life Technologies) and specific TaqMan ${ }^{\circledR}$ probes (Supplementary Table 2). The gene expression levels were normalized to GAPDH.

\section{miRNA quantification}

cDNA was generated from RNA samples using the TaqMan ${ }^{\circledR}$ miRNA reverse transcription kit (Life Technologies). qPCR was performed using cDNA and specific TaqMan ${ }^{\circledR}$ miRNA probes (Supplementary Table 2). The miRNA levels were normalized to U6.

All reactions were performed on a Step-One Plus RTqPCR system (Life Technologies), and a cycle threshold
(Ct) of 0.1 was used to calculate $\Delta \mathrm{Ct}$ values for analysis using the comparative $\Delta \mathrm{Ct}$ method $^{38}$.

\section{Western blotting}

Cells were lysed using radio immune-precipitation assay buffer and protein concentration determined using the bicinchoninic acid assay. The primary antibodies are listed in Supplementary Table 3. Cell lysates were separated by NuPAGE ${ }^{\circledR}$ MOPS SDS, transferred to a nitrocellulose membrane, and developed using a ChemiDoc MP imager (BioRad, Hercules, CA).

\section{Cell proliferation and viability}

HT29, SW480, and Caco2 cells were plated at a concentration of $1 \times 10^{5}$ cells/well in $2 \%$ Fetal Bovine Serum (FBS) supplemented media. The media was changed to $10 \%$ FBS supplemented media after $24 \mathrm{~h}$. Daily cell counts and percentage of viable cells, using Trypan blue, were obtained every $24 \mathrm{~h}$ from 0 to $120 \mathrm{~h}$ using an automatic cell counter (TC20 ${ }^{\mathrm{TM}}$ Bio-Rad Laboratories, Hercules, CA).

\section{Scratch assay}

HT29 and SW480 cells were plated at $1 \times 10^{6}$ cells/well in a 12-well plate. A scratch was made using a sterile $20 \mu \mathrm{l}$ pipette tip. Photos (Nikon Eclipse TS100) were taken every $24 \mathrm{~h}$ from 0 to $120 \mathrm{~h}$ or until complete scratch closure $(x 4$ magnification). The percentage scratch closure for each time point was compared to $0 \mathrm{~h}$.

\section{Transwell migration and invasion}

SW 480 cells were seeded at $2 \times 10^{5}$ cells/well, and HT29 cells were seeded at $5 \times 10^{5}$ cells/well into a transwell migration (COSTAR, $8.0 \mu \mathrm{m}$ pore polycarbonate membrane) or invasion insert (COSTAR, $8.0 \mu \mathrm{m}$ pore Corning BioCoat Matrigel Invasion Chambers), as per the manufacturer's instructions. Complete media (supplemented with $10 \% \mathrm{FBS}$ ) was the chemoattractant, and plates were incubated at $37^{\circ} \mathrm{C}$ for $24 \mathrm{~h}$. Each insert was stained with the Diff-Quik staining kit (Electron Microscopy Sciences, Hatfield, PA), and six representative images were taken ( $\times 10$ magnification). Migrated cells in each field were manually counted.

\section{In vivo tumor growth}

Six-week old male $\mathrm{Nu} / \mathrm{J}$ mice were purchased from the Jackson Laboratory (Bar Harbor, Maine). Each mouse was injected with $5 \times 10^{6}$ cells in $200 \mu \mathrm{L}$ PBS. Mass volume was calculated using the formula: Volume $=0.5 \times$ longitude diameter $\times$ (latitudinal diameter $)^{2}$, every 3 days up to 14 days post mass identification.

\section{Statistical analysis}

All reactions were performed in duplicate, with the average being used for analysis. Data are presented as 
median (interquartile range). The chi-squared test, or Fischer's exact test, were used to compare categorical variables. The Mann-Whitney $U$ test and Wilcoxon signed-rank test were used to compare continuous variables. Differences in overall survival were compared with the Log-rank test. Statistical analysis was performed using SPSS v26.0 (IBM Corp, Armonk, NY). Graphs were created using GraphPad Prism v6.01 (Graphpad Software Inc. La Jolla, CA). Statistical significance was defined as $p<0.05$.

\begin{abstract}
Author details
${ }^{1}$ Price Institute of Surgical Research, Hiram C. Polk Jr. MD Department of Surgery, University of Louisville School of Medicine, Louisville, KY 40202, USA. ${ }^{2}$ Department of Bioinformatics and Biostatistics, University of Louisville, Louisville, KY, USA. ${ }^{3}$ Centre for Agricultural Bioinformatics, ICAR-Indian Agricultural Statistics Research Institute, New Delhi, India. ${ }^{4}$ Department of Veterinary Science, Gluck Equine Research Center, University of Kentucky, Lexington, KY, USA
\end{abstract}

\section{Funding}

This work was supported by the John W. Price and Barbara Thruston Atwood Price Trust and a grant from the Mary K. Oxley Foundation. This work was supported by the University of Louisville Cancer Education Program (NIH 2R25CA134283-06A1) and the American Society for Colon \& Rectal Surgeons Medical Student Research Initiation Grant.

\section{Data availability}

Available upon request

\section{Conflict of interest}

The authors declare no competing interests.

\section{Publisher's note}

Springer Nature remains neutral with regard to jurisdictional claims in published maps and institutional affiliations.

Supplementary information The online version contains supplementary material available at https://doi.org/10.1038/s41420-021-00427-x.

Received: 25 November 2020 Revised: 12 December 2020 Accepted: 3 February 2021

Published online: 26 March 2021

\section{References}

1. Siegel, R. L., Miller, K. D. \& Jemal, A. Cancer statistics. Ca. Cancer J. Clin. 68, 7-30 (2018).

2. Muller, A. D. \& Sonnenberg, A. Prevention of colorectal cancer by flexible endoscopy and polypectomy: a case-control study of 32,702 veterans. Ann. Intern. Med. 123, 904-910 (1995).

3. Kalluri, R. \& Weinberg, R. A. The basics of epithelial-mesenchymal transition. J. Clin. Investig. 119, 1420-1428 (2009).

4. O'Brien, S. J. et al. The role of the miR-200 family in epithelial-mesenchymal transition in colorectal cancer: a systematic review. Int. J. Cancer 142, 2501-2511 (2018)

5. Han, D. et al. Long noncoding RNAs: novel players in colorectal cancer. Cancer Lett. 361, 13-21 (2015).

6. Dong, D., Mu, Z., Zhao, C. \& Sun, M. ZFAS1: a novel tumor-related long noncoding RNA. Cancer Cell. Int. 18, 125 (2018).

7. Bracken, C. P. et al. A double-negative feedback loop between ZEB1-SIP1 and the microRNA-200 family regulates epithelial-mesenchymal transition. Cancer Res. 68, 7846-7854 (2008).

8. Barretina, J. et al. The Cancer Cell Line Encyclopedia enables predictive modelling of anticancer drug sensitivity. Nature 483, 603 (2012).
9. Paraskevopoulou, M. D. et al. DIANA-LncBase v2: indexing microRNA targets on non-coding transcripts. Nucleic Acids Res. 44, D231-D238 (2016).

10. The Cancer Genome Atlas Network. et al. Comprehensive molecular characterization of human colon and rectal cancer. Nature 487, 330 (2012).

11. Paterson, E. L. et al. Down-regulation of the miRNA-200 family at the invasive front of colorectal cancers with degraded basement membrane indicates EMT is involved in cancer progression. Neoplasia 15, 180-191 (2013).

12. Berg, K. C. G. et al. Multi-omics of 34 colorectal cancer cell lines: a resource for biomedical studies. Mol. Cancer 16, 116 (2017).

13. Liu, G. et al. LnCRNA ZFAS1 promotes growth and metastasis by regulating BMI1 and ZEB2 in osteosarcoma. Am. J. Cancer Res. 7, 1450-1462 (2017).

14. Zhang, F. et al. Long non-coding RNA ZFAS1 regulates the malignant progression of gastric cancer via the microRNA-200b-3p/Wnt1 axis. Biosci. Biotechnol. Biochem. 83, 1289-1299 (2019).

15. Xia, B. et al. Long non-coding RNA ZFAS1 interacts with miR-150-5p to regulate Sp1 expression and ovarian cancer cell malignancy. Oncotarget 8, 19534-19546 (2017)

16. Li, T. et al. Amplification of long noncoding rna zfas 1 promotes metastasis in hepatocellular carcinoma. Cancer Res. 75, 3181-3191 (2015).

17. $\mathrm{Wu}$, T. et al. Knockdown of long non-coding RNA-ZFAS1 protects cardiomyocytes against acute myocardial infarction via anti-apoptosis by regulating miR-150/CRP. J. Cell. Biochem. 118, 3281-3289 (2017).

18. Chen, X. et al. SP1-induced IncRNA-ZFAS1 contributes to colorectal cancer progression via the miR-150-5p/NEGFA axis. Cell Death Dis. 9, 982 (2018).

19. Xie, S., Ge, Q., Wang, X., Sun, X. \& Kang, Y. Long non-coding RNA ZFAS1 sponges miR-484 to promote cell proliferation and invasion in colorectal cancer. Cell Cycle 17, 154-161 (2018).

20. Ye, Y., Gao, X. \& Yang, N. LncRNA ZFAS1 promotes cell migration and invasion of fibroblast-like synoviocytes by suppression of miR-27a in rheumatoid arthritis. Hum. Cell 31, 14-21 (2018).

21. O'Brien, S. J. et al. Long non-coding RNA (IncRNA) and epithelial-mesenchymal transition (EMT) in colorectal cancer: a systematic review. Cancer Biol. Ther. 21, 769-781 (2020)

22. Carter, J. V. et al. The microRNA-200 family acts as an oncogene in colorectal cancer by inhibiting the tumor suppressor RASSF2. Oncol. Lett. 18, 3994-4007 (2019).

23. Askarian-Amiri, M. E. et al. SNORD-host RNA Zfas1 is a regulator of mammary development and a potential marker for breast cancer. RNA 17, 878-891 (2011).

24. Fan, S. et al. Downregulation of the long non-coding RNA ZFAS1 is associated with cell proliferation, migration and invasion in breast cancer. Mol. Med. Rep. 17, 6405-6412 (2018)

25. Wang, T. et al. Long noncoding RNA ZNFX1-AS1 suppresses growth of hepatocellular carcinoma cells by regulating the methylation of miR-9. Onco. Targets Ther. 9, 5005-5014 (2016).

26. Lu, T., Wang, Y., Chen, D., Liu, J. \& Jiao, W. Potential clinical application of IncRNAs in non-small cell lung cancer. Onco. Targets Ther. 11, 8045-8052 (2018).

27. Barangi, S., Hayes, A. W., Reiter, R. \& Karimi, G. The therapeutic role of long noncoding RNAs in human diseases: a focus on the recent insights into autophagy. Pharm. Res. 142, 22-29 (2019).

28. Chakraborty, C., Sharma, A. R., Sharma, G., Doss, C. G. P. \& Lee, S. S. Therapeutic miRNA and siRNA: moving from bench to clinic as next generation medicine. Mol. Ther. Nucleic Acids 8, 132-143 (2017).

29. Janssen, H. L. et al. Treatment of HCV infection by targeting microRNA. N. Engl. J. Med. 368, 1685-1694 (2013).

30. Bojmar, L. et al. The role of microRNA-200 in progression of human colorectal and breast cancer. PloS ONE 8, e84815-e84815 (2013).

31. Jahangiri, B., Khalaj-Kondori, M., Asadollahi, E. \& Sadeghizadeh, M. Cancerassociated fibroblasts enhance cell proliferation and metastasis of colorectal cancer SW480 cells by provoking long noncoding RNA UCA1. J. Cell. Commun. Signal 13, 53-64 (2019).

32. $\mathrm{Xu}$, J., Lamouille, S. \& Derynck, R. TGF-beta-induced epithelial to mesenchymal transition. Cell. Res. 19, 156-172 (2009).

33. Cai, J. et al. Tumor-associated macrophages derived TGF- $\beta$-induced epithelial to mesenchymal transition in colorectal cancer cells through Smad2,3-4/Snail signaling pathway. Cancer Res. Treat. 51, 252-266 (2019).

34. O'Brien, S. J., Ekman, M. B., Manek, S. \& Galandiuk, S. CRISPR-mediated gene editing for the surgeon scientist. Surgery 166, 129-137 (2019). 
35. Rankin, C. R. et al. Overexpressing long noncoding RNAs using geneactivating CRISPR. J. Vis. Exp. 145, e59233 (2019). https://doi.org/ 10.3791/59233.

36. McCarthy, D. J., Chen, Y. \& Smyth, G. K. Differential expression analysis of multifactor RNA-Seq experiments with respect to biological variation. Nucleic Acids Res. 40, 4288-4297 (2012).
37. Robinson, M. D., McCarthy, D. J. \& Smyth, G. K. EdgeR: a Bioconductor package for differential expression analysis of digital gene expression data. Bioinformatics 26, 139-140 (2010).

38. Livak, K. J. \& Schmittgen, T. D. Analysis of relative gene expression data using real-time quantitative PCR and the 2(-Delta Delta C(T)) method. Methods 25, 402-408 (2001) 